\title{
Research on Wireless Communication System of Intelligent Home Shiyuan Jia ${ }^{1, a}$ \\ ${ }^{1}$ Beijing University of Posts and Telecommunications, Beijing, China, 102209
}

\author{
Keywords: Wireless Communication System, Intelligent Home, Electricity Device
}

\begin{abstract}
In the mid-1990s, Chinese intelligent building began to develop, from a variety of special features of the intelligent building, in recent years has been facing the construction industry's most basic content - the development of civilian residential. Intelligent home is the inevitable result of the infiltration of IT technology to the traditional construction industry. In order to facilitate the processing, management, transmission and storage of information, the various information nodes (network appliances, security equipment, lighting equipment and environmental control devices) The system bus is connected to the function module, thus forms the home information cable network system. Furthermore, how to manage these network devices humane? Control system is one of the important part of the cable control can not get rid of the shackles of the cable, and wireless control because of its use of space in the flexibility and convenience and it was Widely used in the home environment.
\end{abstract}

\section{Introduction}

The wireless sensor network is a distributed sensor network whose peripherals are sensors that can sense and examine the external world. WSN sensors communicate wirelessly, so the network settings are flexible, the device location can be changed at any time, and can be wired or wirelessly connected to the Internet. Based on low power consumption, self-organization, high reliability, low cost wireless sensor network is the best choice for home wireless network, wireless sensor network can use the existing Internet, mobile communication network and telephone network indoor environment parameters, Home appliances operating status and other information to inform the tenants, so that households can through the internet network to keep abreast of the internal situation, and remote monitoring of home appliances, home and outside the realization of information transmission [1].

\section{Intelligent Home Wireless Control Technology Profile}

IrDA is the abbreviation of Infrared Data Association, the Infrared Data Standards Association, which was established in 1993, is an international standard nonprofit organization dedicated to establishing wireless connectivity. It is a point-to-point data transfer protocol that is the replacement of traditional cable connections between devices. Its communication distance is generally between 0 $\sim 3 \mathrm{~m}$, the transmission rate of up to $16 \mathrm{Mbps}$, the communication medium for the wavelength of 850 $\sim 900 \mathrm{~nm}$ within the near infrared. Infrared anti-interference strong, with the light wave linear propagation characteristics, not easy to produce interference between each other, is a very good information transmission media.

Home RF (HomeRF) is the product of cordless phone technology (digital enhanced cordless phone or referred to as DECT: Digital Enhanced Cordless Tele-phone) and wireless local area network (WLAN) technology integration development. The wireless LAN IEEE802.11 uses CSMA / CA (Carrier Sense Multi-Point Access / Collision Avoidance), which is particularly suitable for data services; and DECT uses TDMA (Time Division Multiplexing), which is particularly suitable for voice communication, (SWAP: Shared Wireless Access Protocol), which forms the home radio frequency. The home RF system is designed to deliver voice and data between appliances and to interact with the public switched telephone network (PSTN) and the Internet. It operates in the 2.4GHz ISM band, using digital frequency hopping spread spectrum technology, data security by Blowfish encryption algorithm to ensure. 
Bluetooth (Bluetooth) technology is a Bluetooth SIG (Special Interest Group) jointly developed by the short-range wireless communication technology standards, the purpose is to achieve the highest data transfer rate of $1 \mathrm{MB} / \mathrm{s}$ (effective data transfer rate is $721 \mathrm{~KB} / \mathrm{s}$ ), the maximum transmission distance 10m wireless communication technology.

Wi-Fi (Wireless Fidelity) is a way to help users access e-mail, Web and streaming media technology. It operates in the 2.4GHZ ISM band and supports speeds up to 54Mbps, providing users with wireless broadband Internet access that can support Internet access within hundreds of feet of radio signals. There are also three 802.11 space protocols, including IEEE 802.11 (a / b / n), which is widely used due to the fact that the actual transmission rate of the $802.11 \mathrm{~g}$ protocol is faster and that the products supporting the protocol are affordable [2].

ZigBee is based on IEEE 802.15.4 wireless standard developed a short distance ( $0 \sim 75 \mathrm{~m})$, low transmission rate (maximum 250Kbps), low power consumption (ordinary alkaline batteries can be maintained for 6 months to 2 years of use) Low complexity, low cost, and high reliability of two-way wireless communication technology. It operates in $2.4 \mathrm{GHz}$ (global), $915 \mathrm{MHz}$ (US) and $868 \mathrm{MHz}$ (Europe) three bands, these three different bands are used phase modulation technology, which 2.4GHz using higher order QPSK modulation technology to achieve 250Kbit / s 16 channels), the higher throughput, smaller communication delay and shorter duty cycle, and thus more power; and $915 \mathrm{MHz}$ and $868 \mathrm{MHz}$, the use of BPSK modulation technology, the transmission rate were 40Kbit / s (10 channels), 20Kbit / s (1 channel), the introduction of these two bands to avoid the $2.4 \mathrm{GHz}$ near the various wireless communication equipment, mutual interference. Unlike the Bluetooth 802.15.1 for voice transmission, Wi-Fi (802.11a / b / g) is suitable for transmitting E-mail and images, ZigBee systems focus on monitoring, control and sensing multi-node network applications.

\section{The System Structure and Design of Intelligent Home}

At present, smart home has been the concern of people, and a large number of smart home products also will be gradually infiltrated into people's lives. At present the indoor installation of intelligent home products usually by the control node and the terminal device composed of two parts. Where the terminal device is directly used to serve the user, interact with the user products, such as television and control nodes are used to control the terminal equipment to produce a response to the needs of users, such as set-top boxes and so on. Among them, the control communication can usually be divided into two parts, respectively, with the terminal equipment to communicate with the Internet and the upper layer of communication, so the control commands for the equipment can be issued indoors, but also through the Internet and other remote control to send the corresponding Equipment.

In the traditional intelligent home system in a variety of applications within the system can achieve the transmission of information, and the future of intelligent home needs between the various devices to connect effectively, so the need for a lot of information transmission, the traditional information transmission are used There are two aspects, one is the need to wiring, installation complexity; Second, when the number of devices in the system continues to increase, the number of transmission lines required also increased, resulting in higher costs. Therefore, the traditional wired data transmission has been unable to meet the requirements, the rise of wireless sensor networks.

The smart home system has space, cost and real-time constraints, where the choice of embedded technology to design intelligent home gateway, to improve the utilization of resources. Smart home gateway hardware design structure intelligent home gateway hardware architecture is based on the embedded processor chip, power and reset the configuration circuit, ZigBee wireless communication module, wired network interface, wireless network module, input and output devices , Serial and other necessary peripherals, for the software to provide communication interface and platform. ZigBee wireless network formed a home internal network, responsible for collecting home internal environment information; GPRS module to achieve intelligent home remote monitoring; the same time, LCD is used to display the user management interface, the 
coordinator through the RS232 serial port and the gateway main controller connected.

\section{Intelligent Home System Software Design}

Intelligent home gateway mainly to achieve the following functions: the completion of the internal sensor nodes within the communication, and control of the nodes; to achieve internal network and external network information exchange, the face of more communication tasks and network interconnection, the need to select the appropriate embedded Type operating system to debug and run, so as to improve the overall efficiency of the system. This article chooses Win CE operating system to complete the embedded home gateway.

Establish a network in the actual network construction process each coordinator can establish a network, if there is a ready-made network coordinator can request to join the network, if not on their own to establish their own network. The coordinator must set some important parameters before the network is established, including the channel that the network is working on, whether or not to use beacon mode. The specific process of network establishment is to call the MAC layer of the primitive has the ability to scan and the main scan.

Nodes join a network If the coordinator is meaningless, more nodes join the network, the coordinator can play a greater role [3]. The following will explain in detail how the nodes join the network. The joining of the nodes includes two aspects: how the new nodes discover the existing networks and join them, and how the existing network nodes allow other nodes to join. Nodes join the network in a variety of ways, through the connection to join the network, re-join the network, isolated nodes to join the network and so on.

The temperature sensor node design is used in the component DS18B20, which is a very simple component of the hardware, from the front of the content we know it is generally only three pins, so when the hardware is connected, only the three pins need to be connected correctly To CC2530 corresponding to the three pins on it, so that a complete temperature sensor node to build a successful [4]. Then the software design of the temperature sensor node, the software design includes the initialization of various functional modules, interrupt handling and read and write DS18B20 temperature detection program. The initialization program includes the system main clock source setting, analog-to-digital conversion initialization, serial port initialization, clock mode initialization, advanced encryption standard initialization, RF initialization, interrupt initialization and DS18B20 initialization. It should be noted that if a plurality of temperature sensor nodes are placed inside the home, the single point temperature measurement becomes a multi-point temperature measurement. Since multiple temperature sensor nodes are connected to a bus, the user does not know which sensor node the temperature information is from if it is not marked. Therefore, to identify different nodes, before the system is installed, the home gateway should be connected with the temperature sensor and set the serial number for it. The specific operation is the intelligent gateway to the temperature sensor to send a reset pulse, the temperature sensor to respond to the response pulse, the home gateway to send ROM command, and send a pulse about 2us, and then read the temperature sensor serial number of a bit, and the same Method reads every bit of the serial number [5].

\section{Conclusion}

With the rapid development of things, smart home has gradually become a reality. As a result of the intelligent home, it is in a number of devices will be interconnected, so the need for massive data transmission [6]. On this basis, wireless sensor network technology has been rapid development including wireless communication technology is an important part. In this paper, the commonly used short-range wireless communication technology research and comparison, summed up their advantages and disadvantages, and on this basis to discuss its application in the smart home. In the use of the process, how to integrate different communication technology, enhance the transmission rate of wireless transmission signal, security and stability is the main application in the future research. 


\section{References}

[1] Smart home to create a smart life [J]. Intelligent Building, 2015 (01)

[2] Wang Fei, Chen Jinying, Liu Xiangyan. Intelligent home application status and development analysis [J]. Communication world, 2016 (04)

[3] Hao Hailing. Intelligent home technology development and application of thinking [J]. Inner Mongolia Science and Technology and Economy, 2016 (08)

[4] Zhang Yan. Chinese smart home status and development direction [J]. Shanxi Electronic Technology, 2016 (04)

[5] Liang Guoqin. Pursuit of my ideal "4S" smart home [J]. Intelligent building, 2013 (02)

[6] Feng Kai, Tong Shihua. The origin of smart home and its development trend [J]. Chinese new technology and new products, 2010 (06) 